\title{
Stem cells and neurodegenerative diseases: where is it all going?
}

\author{
Roger A Barker:
}

Over the last few years there have been a number of major breakthroughs in the development of stem cells for diseases of the CNS. One of these has been in the ability to reprogram adult somatic cells to a more pluripotent state as well as directly to neurons and, by so doing, use patient-derived cells to study disease. In addition, the capacity to engineer embryonic stem cells to defined neuronal fates in the absence of proliferative contaminant cells is now feasible, which opens up the possibility of using these cells for cell transplantation. In this review, we will discuss how these developments have come about, particularly in the context of Parkinson's disease, and what this means for the future of this whole field over the next few years.

KEYWORDS: - induced pluripotent stem cells - neural grafting - Parkinson's disease a stem cells

Chronic neurodegenerative disorders of the CNS, which target the aging brain, are set to increase as the population ages. As such, finding ways to better understand and treat these conditions is a major challenge given the personal and economic costs. These disorders include Alzheimer's disease (AD), Parkinson's disease (PD) and a host of other rarer conditions, such as Huntington's disease (HD), frontotemporal dementia and amyotrophic lateral sclerosis. Each of these disorders is defined by the loss of specific populations of neurons, with a characteristic pathological pattern of protein aggregation - for example, in the case of PD the loss of the nigrostriatal dopaminergic pathway and the presence of alpha synuclein-containing Lewy bodies.

While this is a useful starting point by which to define these diseases, it is nevertheless important to realize that these chronic neurodegenerative disorders:

- Have a much greater extent of pathological burden than was once recognized and as such these diseases

\footnotetext{
*Author for correspondence: Roger A Barker, Cambridge Centre for Brain Repair Department of Clinical Neuroscience, University of Cambridge, Forvie Site,

Robinson Way, Cambridge CB2 OPY, UK; Tel.: +44 1223331 160; Fax: +44 1223331

174; rab46@cam.ac.uk
} one neuronal network; and an inflammatory element; affect the more elderly; etiology; neurogenic processes. target a whole range of different neuronal populations, rather than just

- Have a pathology that is not confined to the neurons but involves glial cells

- Often display mixed profiles of pathology, typically with a significant vascular disease burden in the brain, particularly in those conditions that

- Are heterogeneous with a complex

- May have an effect on endogenous

Viewing these disorders as a discrete entity with a single pathology and core cell loss has been a useful first approximation, which has to date been best modelled using either targeted lesions (e.g., a lesion to the dopaminergic nigrostriatal neurons in the case of $\mathrm{PD})$ or the generation of a transgenic murine model if the condition had a known Mendelian basis. While these models are useful, they provide only limited information as the majority of patients with the most common neurodegenerative conditions have a non-Mendelian form of the condition with a complex evolving pathological process across networks of cells. However, the newly developed capacity to reprogram adult somatic cells from patients with these diseases has opened up new possibilities in this area. In this short review, the author will discuss how this technology of inducible stem cells has been used to better understand these diseases and how stem cells are being viewed for cell transplantation.

Disease modeling: the use of inducible cells

Using stem cells to dissect the cellular events underlying the genesis of different neurodegenerative conditions has gained prominence following the seminal demonstration that differentiated human somatic cells could be reprogrammed into a pluripotent state by the overexpression of a set of defined transcription factors $[1,2]$. This technology using induced pluripotent stem (iPS) cells means that cells can be grown from patients themselves that have the capacity to proliferate indefinitely in culture and a pluripotency profile that enables them 


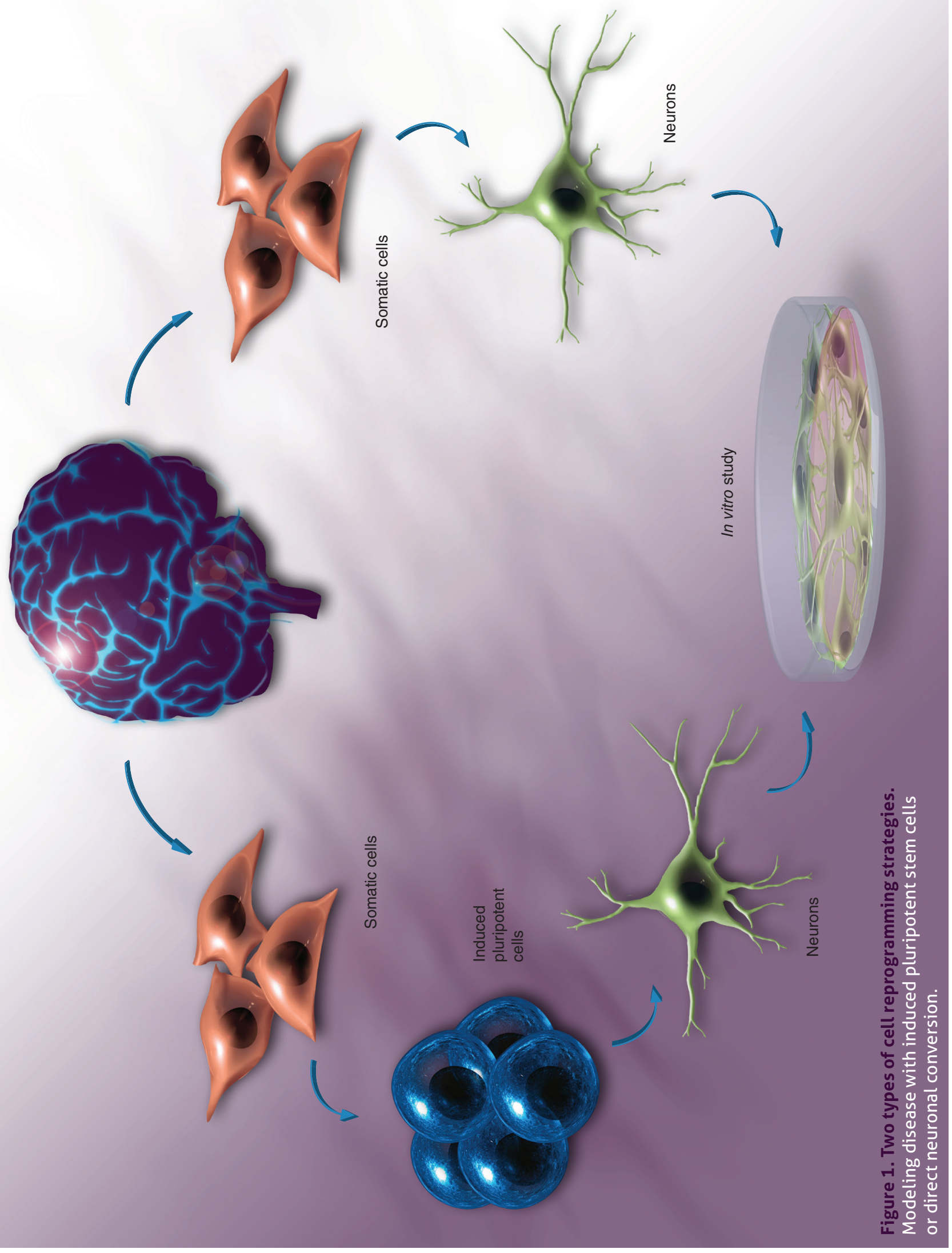


to be differentiated into any cell type, including different types of neurons.

More recently it has been shown that it is possible to directly reprogram human somatic cells into neurons without having to go through an intermediate pluripotent state [3]. Again these reprogrammed induced neurons could be extremely useful for screening potential compounds for therapeutic purposes, as well as studying disease pathways.

So what has been shown with these two types of cell reprogramming strategies (FIGURE 1), and what has this told us about neurodegenerative disorders?

\section{- Modeling disease with iPS cells} iPS cells derived from patients offer a powerful in vitro model by which to study disease, as these cells carry all of the necessary genetic risk factors for that disorder. The ability to make such cell lines and derive relevant neurons from them has now been achieved in a range of conditions but mainly from patients with Mendelian forms of disease such as some types of PD (see below) and AD, as well as HD and some genetic forms of motorneuron disease and dementia [4-8]. These studies have largely used fibroblasts harvested from skin biopsies and a variety of different reprogramming and culturing regimes - techniques that are constantly being refined and improved upon.

These studies have drawn a number of important conclusions about the use of iPS cells to model disease. A key finding is that relevant populations of neurons can be derived using this approach. Importantly though, it is increasingly being recognized that such cells need to be shown to be true neurons of the type relevant for the disease state (e.g., dopaminergic nigral neurons in the case of PD). In other words, the character of the derived neurons needs to be verified using not just immuncytochemistry but transcription profiles, along with evidence of electrical excitability and transmitter release.
The neurons derived using this approach often have a subtle pathology, if any. This can be brought out by increasing the time spent in culture and/or the use of stressful stimuli. For example, Soldner and colleagues found no differences between the dopaminergic neurons derived from the iPS cells from PD patients and controls after 30 days in culture even though they showed that the cells had a true ventral midbrain dopaminergic neuronal phenotype $[9,10]$. However, long-term culturing ( $<75$ days) of these cells did reveal changes, with a decrease in the number and length of neurites, and an increased susceptibility to degeneration and abnormalities in autophagosome function [10].

Another issue in modeling diseases using somatic cells is the variable biological characteristics of the cells, which include differences in genetic background, as well as in the cell derivation and differentiation processes $[9,11,12]$, along with the genetic alterations introduced during the reprogramming process [13-15]. All of this means that it is unclear whether deficits found in the cells truly relate to the disease state rather than the person or the derivation procedure itself.

In cases of diseases caused by a single abnormal gene, this can be controlled for. For example, Soldner and colleagues (2011) generated human iPS cell lines from a patient carrying the A53T (G209) mutation in the SNCA gene, which they then corrected using zinc finger nuclease-mediated genome editing [16]. By so doing, they generated iPS cells that differ only in this gene (i.e., a gene that gives the cell its susceptibility for PD), providing genetically matched control cells to study the effects of that specific mutation. However, while this approach is appealing to study cellular mechanisms associated with Mendelian forms of disease, it excludes the use of iPS cell lines to investigate disorders with a more complex and mixed etiology, such as most cases of $\mathrm{AD}$ and $\mathrm{PD}$.
A further issue is whether iPS cells can truly be used to study pathologies found mainly in the aged CNS, such as in PD or AD? The induction of pluripotency is accompanied by a progressive elongation of telomeres with passaging [17-19], thus rejuvenating the cells in a similar way to that seen in the embryonic stem (ES) cell state, even in cells derived from aged individuals. However, the telomere chromatin does return to a more mature state when differentiated and iPS cells also retain the DNA methylation patterns of their original state before derivation [20-22], all of which argues for them still being relevant as an in vitro model for these disorders.

Finally, most diseases of the CNS are now known to have multiple players, such that disease pathogenesis involves not just neurons but glia, as well as inflammatory cells. The ability to model disease using the conversion of somatic cells from patients would therefore benefit from the development of cocultures of multiple induced cell lineages. In this respect, the ability to make these different cell types and even induced neural stem cells from fibroblasts would be useful [23-26]. In terms of the inflammatory components and the microglia, such cells have been generated from mouse ES cells [27], but the successful reprogramming of somatic cells, such as fibroblasts, into this cell type has yet to be reported.

\section{Modeling disease with direct neuronal conversion}

While the use of iPS cells has shown great promise as a way to study disease in vitro, concerns with respect to their utility to do this have arisen (see above) in part because they are reprogrammed back to a more pluripotent stage. To overcome this issue, several groups have developed methods that allow direct conversion of human differentiated somatic cells, such as fibroblasts, into functional neurons, avoiding a pluripotent state [3].

The first proof-of-concept study converted mouse embryonic and 
postnatal fibroblasts into functional induced neurons by the overexpression of three transcription factors (Ascl1, Brn2 and Mytl1). These induced neurons displayed appropriate neuronal properties such as the generation of action potentials as well as synapse formation [3]. Human fibroblasts have also now been successfully converted into functional neurons by again overexpressing the same transcription factors [28], and several subsequent studies have been undertaken to better optimize this approach [29,30]. To date, however, its use in patients is limited in so much as this approach has only been done in AD patients with the generation of functional glutamatergic forebrain neurons from fibroblasts [31].

As the direct conversion does not go through a proliferative state, the quantity of neurons that can be obtained is limited by the number of fibroblasts used as the starting material for conversion and thus, to some, these cells are a less attractive tool by which to study disease.

\section{Stem cell transplants}

Although a number of diseases have been thought suitable for this approach (e.g., HD [32]), the one condition where this has been most explored is PD [33]. While a number of different cell types have been tried to restore the dopaminergic deficits that lie at the heart of PD, the most successful approach to date involves the transplantation of fetal ventral mesencephalic (VM) tissue into the striatum of patients, although the results from this approach have been somewhat inconsistent [34-36]. These studies, which at most have involved a few hundred patients, have shown that VM dopaminergic neurons can survive long term in the PD brain and produce significant functional benefits in some patients [33,36], implying that nigral dopaminergic neurons derived from stem cells sources should, theoretically, have the same capabilities.

The differentiation of human ES cells into dopaminergic neurons using standard protocols has long been known to be inefficient, but by using combinations of factors known to be involved in normal dopaminergic neuronal development, the yield of dopaminergic cells from such sources can be dramatically improved $[37,38]$. This ability to make large numbers of functional nigral dopaminergic cells from ES cell sources without teratoma formation has been shown in a number of in vivo transplant studies, although the results until recently were less impressive than older studies using fetal VM tissue [39]. However, a very recent and novel approach from the laboratory of Lorenz Studer has shown that dopaminergic neurons derived from a human ES cell source can have robust effects across a range of animal models of PD [40], which has raised the very real possibility that dopaminergic neurons derived from an ES cell source may be ready for early clinical trials in patients with PD in the next 5 years.

Although the use of ES cells is very promising, there are still concerns with their use, including ethical, immunological and practical issues with their derivation, and as a result iPS and induced neuron cells have been considered as alternatives for grafting. This approach has been assessed in a number of studies, and it has been shown that while it is possible to make functionally relevant nigral dopaminergic neurons from such sources, there are still issues of cellular proliferation within the graft, as well as limited fiber outgrowth and incomplete functional recovery after grafting [41]. In addition, these cells may not be as immunogenically silent as once thought [42].

More recently, directly converted induced dopaminergic neurons from mouse fibroblasts have been transplanted into 6-OHDA-lesioned rodents [43,44], with some success, although again their ability to do this seems less than that seen with fetal VM tissue.

Therefore, although several proof-ofconcept studies have been undertaken to show that it is possible to make functional dopaminergic neurons from iPS (and to a lesser extent induced neurons) cells, a number of questions remain to be answered before they can be considered for use in any clinical transplant trial:

Q Do these cells really form mature nigral dopaminergic neurons with the necessary axonal outgrowth and arborization required of them to innervate the whole striatum when grafted?

Q How safe are these cells in terms of cell proliferation, dedifferentiation, cell migration and the immune reaction they induce?

Q Do these cells retain a disease-specific vulnerability that will adversely affect their long-term survival and efficacy?

Q In the case of Mendelian forms of disease (including PD), can we repair disease-related mutations in vitro and transplant these cells successfully?

O. Can these cells be reprogrammed to replace all of the cell losses seen in this disease?

\section{Conclusion}

The ability to use stem cells to study neurodegenerative diseases has received much attention of late, but it is still not clear whether these cells can truly recapitulate the pathogenic processes in these disorders. It may be that they are ultimately better for doing this in neurodevelopmental disorders of the CNS [45-47]. Nevertheless, the ability to manipulate adult somatic cells from patients with neurodegenerative disorders of the aging CNS to neuronal phenotypes of relevance is now possible, and this may open up exciting new opportunities for cell grafting - although such a therapy would have to be shown to be competitive with the other treatments that are out there, and this includes the cost. However, more realistically it is in the realm of disease modeling and drug screening that they offer most hope, especially given the distributed neuronal pathology seen in all neurodegenerative disorders of the CNS. 
Financial \& competing interests

disclosure

The author has no relevant affiliations or financial involvement with any organization or entity with a financial interest in or financial conflict with the subject matter or materials discussed in the manuscript. This includes employment, consultancies, honoraria, stock ownership or options, expert testimony, grants or patents received or pending, or royalties.

No writing assistance was utilized in the production of this manuscript.

\section{Key points}

- Parkinson's disease (PD) is a disorder that has widespread pathology and clinical features with a core loss of the nigral dopaminergic projection neurons.

- Many Mendelian forms of PD have now been described, and most of these have now been the subject of induced pluripotent stem cell studies. These studies have revealed subtle abnormalities, if any, in the dopaminergic neurons derived from such induced pluripotent stem cells, but they are increasingly being used to study disease pathogenesis and for drug screening.

- It is now possible to make nigral dopaminergic neurons directly from skin fibroblasts, although their capacity to repair the brain after grafting is not known.

- Embryonic stem cell-derived dopaminergic neurons can be generated that have long-term functional benefits in animal models of PD without problems of tumor formation or cellular overgrowth.

- The ability to translate these new stem cell-based therapies into early trials in patients with PD is still some way off, but lessons learnt from the clinical fetal ventral mesencephalic studies may be instructive in this process.

\section{References}

Papers of special note have been highlighted as:

"n of considerable interest

1 Takahashi K, Tanabe K, Ohnuki M et al. Induction of pluripotent stem cells from adult human fibroblasts by defined factors. Cell 131, 861-872 (2007).

2 Yu J, Vodyanik MA, Smuga-Otto K et al. Induced pluripotent stem cell lines derived from human somatic cells. Science 318, 1917-1920 (2007).

3 Vierbuchen T, Ostermeier A, Pang ZP et al. Direct conversion of fibroblasts to functional neurons by defined factors. Nature 463, 1035-1041 (2010).

4 The HD iPSC Consortium. Induced pluripotent stem cells from patients with Huntington's disease show CAG-repeatexpansion-associated phenotypes. Cell Stem Cell 11(2), 264-278 (2012).

5 Dimos JT, Rodolfa KT, Niakan KK et al. Induced pluripotent stem cells generated from patients with ALS can be differentiated into motor neurons. Science 321, 1218-1221 (2008).

6 Hu BY, Zhang SC. Differentiation of spinal motor neurons from pluripotent human stem cells. Nat. Protoc. 4 , 1295-1304 (2009).

7 Park IH, Arora N, Huo H et al. Diseasespecific induced pluripotent stem cells. Cell 134, 877-886 (2008).

8 Bilican B, Serio A, Barmada SJ et al. Mutant induced pluripotent stem cell lines recapitulate aspects of TDP-43 proteinopathies and reveal cell-specific vulnerability. Proc. Natl Acad. Sci. USA 109 (15), 5803-5808 (2012).

9 Soldner F, Hockemeyer D, Beard C et al. Parkinson's disease patient-derived induced pluripotent stem cells free of viral reprogramming factors. Cell 136, 964-977 (2009).

10 Sanchez-Danes A, Richaud-Patin Y, Carballo-Carbajal I et al. Disease-specific phenotypes in dopamine neurons from human iPS-based models of genetic and sporadic Parkinson's disease. EMBO Mol. Med. 4, 380-395 (2012).

11 Ohi Y, Qin H, Hong C, Blouin L et al. Incomplete DNA methylation underlies a transcriptional memory of somatic cells in human iPS cells. Nat. Cell Biol. 13, 541-549 (2011).

12 Bock C, Kiskinis E, Verstappen G et al. Reference maps of human ES and iPS cell variation enable high-throughput characterization of pluripotent cell lines. Cell 144, 439-452 (2011).

13 Boulting GL, Kiskinis E, Croft GF et al. A functionally characterized test set of human induced pluripotent stem cells. Nat. Biotechnol. 29, 279-286 (2011).

14 Gore A, Li Z, Fung HL, Young JE et al. Somatic coding mutations in human induced pluripotent stem cells. Nature 471, 63-67 (2011).

15 Hussein SM, Batada NN, Vuoristo S et al. Copy number variation and selection during reprogramming to pluripotency. Nature 471, 58-62 (2011).
16 Soldner F, Laganiere J, Cheng AW et al. Generation of isogenic pluripotent stem cells differing exclusively at two early onset Parkinson point mutations. Cell 146, 318-331 (2011).

17 Marion RM, Strati K, Li H, Tejera A et al. Telomeres acquire embryonic stem cell characteristics in induced pluripotent stem cells. Cell Stem Cell 4, 141-154 (2009).

18 Mathew R, Jia W, Sharma A et al. Robust activation of the human but not mouse telomerase gene during the induction of pluripotency. FASEB J. 24, 2702-2715 (2010).

19 Suhr ST, Chang EA, Rodriguez RM et al. Telomere dynamics in human cells reprogrammed to pluripotency. PLoS ONE 4, e8124 (2009).

20 Yehezkel S, Rebibo-Sabbah A, Segev Y et al. Reprogramming of telomeric regions during the generation of human induced pluripotent stem cells and subsequent differentiation into fibroblast-like derivatives. Epigenetics 6, 63-75 (2011).

21 Hewitt KJ, Shamis Y, Hayman RB et al. Epigenetic and phenotypic profile of fibroblasts derived from induced pluripotent stem cells. PLoS ONE 6, e17128 (2011).

22 Kim K, Doi A, Wen B et al. Epigenetic memory in induced pluripotent stem cells. Nature 467, 285-290 (2010).

23 Emdad L, D'Souza SL, Kothari HP et al. Efficient differentiation of human embryonic and induced pluripotent stem 
cells into functional astrocytes. Stem Cells Dev. 21, 404-410 (2012).

24 Krencik R, Weick JP, Liu Y et al. Specification of transplantable astroglial subtypes from human pluripotent stem cells. Nat. Biotechnol. 29, 528-534 (2011).

25 Krencik R, Zhang SC. Directed differentiation of functional astroglial subtypes from human pluripotent stem cells. Nat. Protoc. 6, 1710-1717 (2011).

26 Thier M, Worsdorfer P, Lakes YB et al. Direct conversion of fibroblasts into stably expandable neural stem cells. Cell Stem Cell 10, 473-479 (2012).

- First paper to describe the direct conversion of adult cells into neural precursor cells and how this could then be used for deriving further cell types.

27 Beutner C, Roy K, Linnartz B et al. Generation of microglial cells from mouse embryonic stem cells. Nat. Protoc. 5 , 1481-1494 (2010).

28 Pfisterer U, Kirkeby A, Torper O et al. Direct conversion of human fibroblasts to dopaminergic neurons. Proc. Natl Acad. Sci USA 108, 10343-10348 (2011).

- First paper to describe the direct conversion of adult human fibroblasts into nigral dopaminergic neurons.

29 Ambasudhan R, Talantova M, Coleman R et al. Direct reprogramming of adult human fibroblasts to functional neurons under defined conditions. Cell Stem Cell 9, 113-118 (2011).

30 Yoo AS, Sun AX, Li L et al. MicroRNAmediated conversion of human fibroblasts to neurons. Nature 476, 228-231 (2011).

31 Qiang L, Fujita R, Yamashita T et al. Directed conversion of Alzheimer's disease patient skin fibroblasts into functional neurons. Cell 146, 359-371 (2011).
2 Wijeyekoon R, Barker RA. The current status of neural grafting in the treatment of Huntington's disease. A review. Front. Integr. Neurosci. 5, 78 (2011).

33 Brundin P, Barker RA, Parmar M. Neural grafting in Parkinson's Disease. Problems and possibilities. Prog. Brain Res. 184, 265-294 (2010).

-1. Useful summary of all of the results and issues arising from the different transplant studies in Parkinson's disease.

34 Freed CR, Greene PE, Breeze RE et al. Transplantation of embryonic dopamine neurons for severe Parkinson's disease. N. Engl. J. Med. 344(10), 710-719 (2001).

35 Olanow CW, Goetz CG, Kordower JH et al. A double-blind controlled trial of bilateral fetal nigral transplantation in Parkinson's disease. Ann. Neurol. 54(3), 403-414 (2003).

36 Politis M, Wu K, Loane C et al. Serotonergic neurons mediate dyskinesia side effects in Parkinson's patients with neural transplants. Sci. Transl. Med. 2(38), 38ra46 (2010).

37 Sanchez-Danes A, Consiglio A, Richaud Y et al. Efficient generation of A9 midbrain dopaminergic neurons by lentiviral delivery of LMX1A in human embryonic stem cells and induced pluripotent stem cells. Hum. Gene Ther. 23, 56-69 (2012).

38 Kim H, Lee G, Granat Y et al. miR-371-373 expression predicts neural differentiation propensity in human pluripotent stem cells. Cell Stem Cell 8(6), 695-706 (2011).

39 Roy NS, Cleren C, Singh SK et al. Functional engraftment of human ES cellderived dopaminergic neurons enriched by coculture with telomerase-immortalized midbrain astrocytes. Nat. Med. 12(11), 1259-1268 (2006).
40 Kriks S, Shim JW, Piao J et al. Dopamine neurons derived from human ES cells efficiently engraft in animal models of Parkinson's disease. Nature 480, 547-551 (2011).

- Breakthrough paper in which nigral dopaminergic neurons were made from human embryonic stem cells using a developmental approach leading to cells with functional capacity and no tumor formation.

41 Hargus G, Cooper O, Deleidi M et al. Differentiated Parkinson patient-derived induced pluripotent stem cells grow in the adult rodent brain and reduce motor asymmetry in Parkinsonian rats. Proc. Natl Acad. Sci. USA 107, 15921-15926 (2010).

42 Zhao T, Zhang ZN, Rong Z, Xu Y. Immunogenicity of induced pluripotent stem cells. Nature 474, 212-215 (2011).

43 Kim J, Su SC, Wang H et al. Functional integration of dopaminergic neurons directly converted from mouse fibroblasts. Cell Stem Cell 9, 413-419 (2011).

44 Liu X, Li F, Stubblefield EA et al. Direct reprogramming of human fibroblasts into dopaminergic neuron-like cells. Cell Res. 22, 321-332 (2012).

45 Farra N, Zhang WB, Pasceri P et al. Rett syndrome induced pluripotent stem cellderived neurons reveal novel neurophysiological alterations. Mol. Psychiatry doi:10.1038/mp.2011.180 (2012) (Epub ahead of print).

46 Pedrosa E, Sandler V, Shah A et al. Development of patient-specific neurons in schizophrenia using induced pluripotent stem cells. J. Neurogenet. 25, 88-103 (2011).

47 DeRosa BA, Van Baaren JM, Dubey GK et al. Derivation of autism spectrum disorder-specific induced pluripotent stem cells from peripheral blood mononuclear cells. Neurosci. Lett. 516, 9-14 (2012). 